\title{
Determination of Gum Arabic (Acacia nilotica) Constituents Using Laser Induced Breakdown Spectroscopy
}

\section{Mashair Ahmed Mohammed Yousif ${ }^{1}$, Nafie A. Almuslet ${ }^{2}$, Elfatih A. Hassan ${ }^{3}$, Fatima Medany Mohamed Taha ${ }^{4}$, Mohammed Idriss Ahmed Mohammed ${ }^{3}$, Abeer Eltaybe Fagir Osman ${ }^{5}$}

\begin{abstract}
${ }^{1}$ College of Science in Alkhurma, Taif University, Alkhurma, Saudi Arabia; ${ }^{2}$ Almogran College of Science and Technology, Khartoum, Sudan; ${ }^{3}$ Sudan University of Science and Technology, Khartoum, Sudan; ${ }^{4}$ College of Science, University of Khartoum, Khartoum, Sudan; ${ }^{5}$ College of Education, Qassim University, Osman, KSA
\end{abstract}

Correspondence to: Mashair Ahmed Mohammed Yousif, garaof@yahoo.com

Keywords: Gum Arabic, LIBS, Laser Spectroscopy, Gum Investigation

Received: February 15, $2021 \quad$ Accepted: March 26, $2021 \quad$ Published: March 29, 2021

Copyright $\odot 2021$ by author(s) and Scientific Research Publishing Inc.

This work is licensed under the Creative Commons Attribution International License (CC BY 4.0).

http://creativecommons.org/licenses/by/4.0/

\section{(c) (i) Open Access}

\section{ABSTRACT}

In this work, Laser Induced Breakdown Spectroscopy (LIBS) was used to determine the constituent of Gum Arabic (Acacia nilotica) collected from five different locations in Sudan. Gum samples were irradiated with $80 \mathrm{~mJ}$ pulse energy of Nd-YAG laser (1064 $\mathrm{nm}$ ) and Atomic spectra Database was used for the spectral analysis of the plasma emitted from these samples. It was found that the samples contain the elements $\mathrm{C}, \mathrm{O}, \mathrm{H}, \mathrm{S}, \mathrm{N}, \mathrm{P}, \mathrm{Na}, \mathrm{Mg}, \mathrm{Ca}$, $\mathrm{Fe}, \mathrm{Cr}, \mathrm{Mn}$, Co with different amounts. Some elements like ( $\mathrm{Ti}, \mathrm{Br}, \mathrm{Ar}, \mathrm{Th}, \mathrm{Kr}, \mathrm{Sc}$ and $\mathrm{Pr}$ ) are recorded here for the first time.

\section{INTRODUCTION}

Laser-induced breakdown spectroscopy (LIBS) is a method of atomic emission spectroscopy (AES) that uses laser-generated plasma as the hot vaporization, atomization, and excitation source [1-4]. Foremost of these is the ability to interrogate samples in situ and remotely without any preparation. In its basic form, a LIBS measurement is carried out by forming laser plasma on or in the sample and then collecting and spectrally analyzing the plasma light. Qualitative and quantitative analyses are carried out by monitoring emission lines positions and intensities [5-7]. LIBS can be used to investigate different materials especially those composed of large molecules such as Gum Arabic. Acacia nilotica Sunt has been found the most valuable timber-producing species [8-10]. An ability to regenerate successfully on flooded sites along the Nile and its tributaries, coupled with timber properties that satisfy most of the utilization standards make the species the most important in the economy of the Sudan. This work aimed to use LIBS for Identification of Gum Arabic (Acacia nilotica). 


\section{METHODS AND MATERIAL}

\subsection{Experimental Setup}

Figure 1 illustrates the LIBS setup which was used in this work. The LIBS system composed of Q-switched $\mathrm{Nd}-\mathrm{YAG}$ Laser (Laser wavelength is $1064 \mathrm{~nm}$, pulse duration $10 \mathrm{~ns}$, Pulse Energy $80 \mathrm{mj}$, Spot size 2 - 8 $\mathrm{mm}$, and repetition rate $2 \mathrm{~Hz}$ ), Ocean Optics $4000+$ spectrometer, connected with PC.

\subsection{The Materials}

Four samples of GumArabic (Acacia nilotica) (sunt) obtained from different locations in Sudan. Were used in this work, they are illustrated in Table 1.

\subsection{Experimental Procedure}

Each sample was put in a quartz cell and irradiated by the Nd-YAG laser where the spark of the sample plasma was collected by a fiber optic to the spectrometer which was interfaces to a computer. The emission spectra were collected in the range from $200-900 \mathrm{~nm}$. In order to test the homogeneity of Gum Arabic samples, several LIBS measurements were performed at its surface. The recorded spectra of the samples were analyzed using Atomic Spectral Database (NIST) data.

Irradiation with pulse energy of $80 \mathrm{~mJ}$ resulted in generating exited elemental species and cations in higher oxidation states. This was evident from the emission spectral lines corresponding to species such as $\mathrm{Cr}^{3+}, \mathrm{Ti}^{2+}$ and $\mathrm{Ar}^{3+}$.

Although the samples of GA in this study were collected from different locations in Sudan having different soil characteristics, their LIBS emission spectra reflect presence of the same elements in all samples. This may indicate that there is no influence of the soil type on the elemental composition of exudates gums like GA.

\section{RESULTS AND DISCUSSION}

LIBS emission spectra of Acacia nilotica (sunt)gum samples irradiated with laser of $80 \mathrm{~mJ}$ pulse energy are shown in Figures 2-5 while Table 2 shows the results of analysis and elemental composition of the mentioned gum samples, which were obtained with the help of Atomic spectra database and the Handbook of basic Atomic spectroscopy.

The elements constituting the samples observed in the emission spectra were $\mathrm{C}, \mathrm{O}, \mathrm{H}, \mathrm{S}, \mathrm{N}, \mathrm{P}, \mathrm{Na}$, $\mathrm{Mg}, \mathrm{Ca}, \mathrm{Fe}, \mathrm{Cr}, \mathrm{Mn}$, Co. This is in agreement with findings of other researches published previously. [11-13]. In addition to these, other elements had been detected for the first time in natural gums, namely

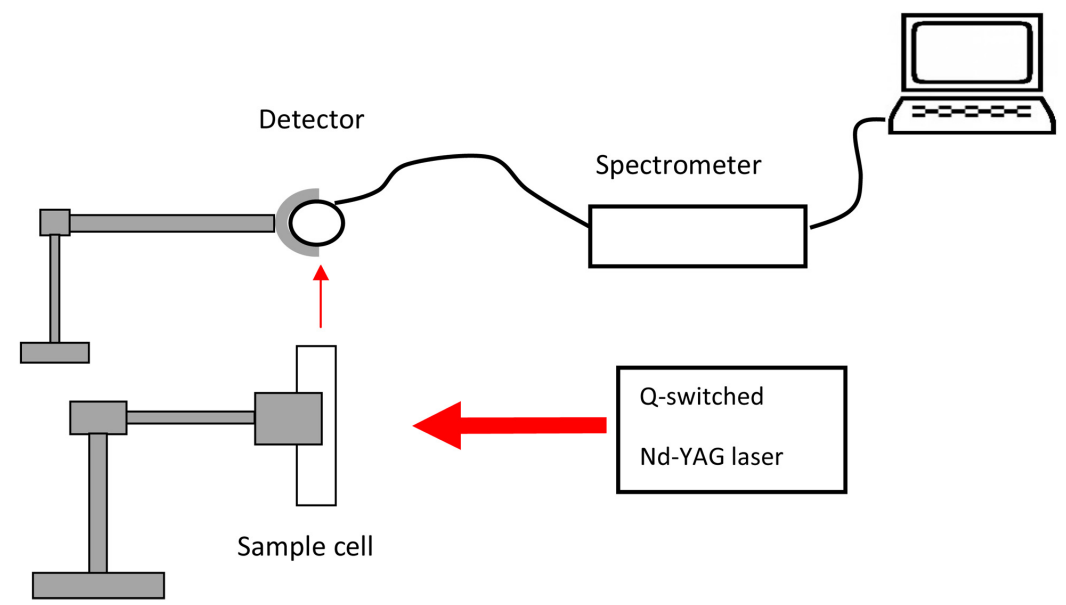

Figure 1. Schematic diagram of the setup. 
Table 1. Samples grouping.

\begin{tabular}{cc}
\hline Classification & Location of samples collection \\
\hline Sample (1) & South Kordofan \\
Sample (2) & Blue Nile state \\
Sample (3) & White Nile state \\
Sample (4) & Khartoum state \\
\hline
\end{tabular}

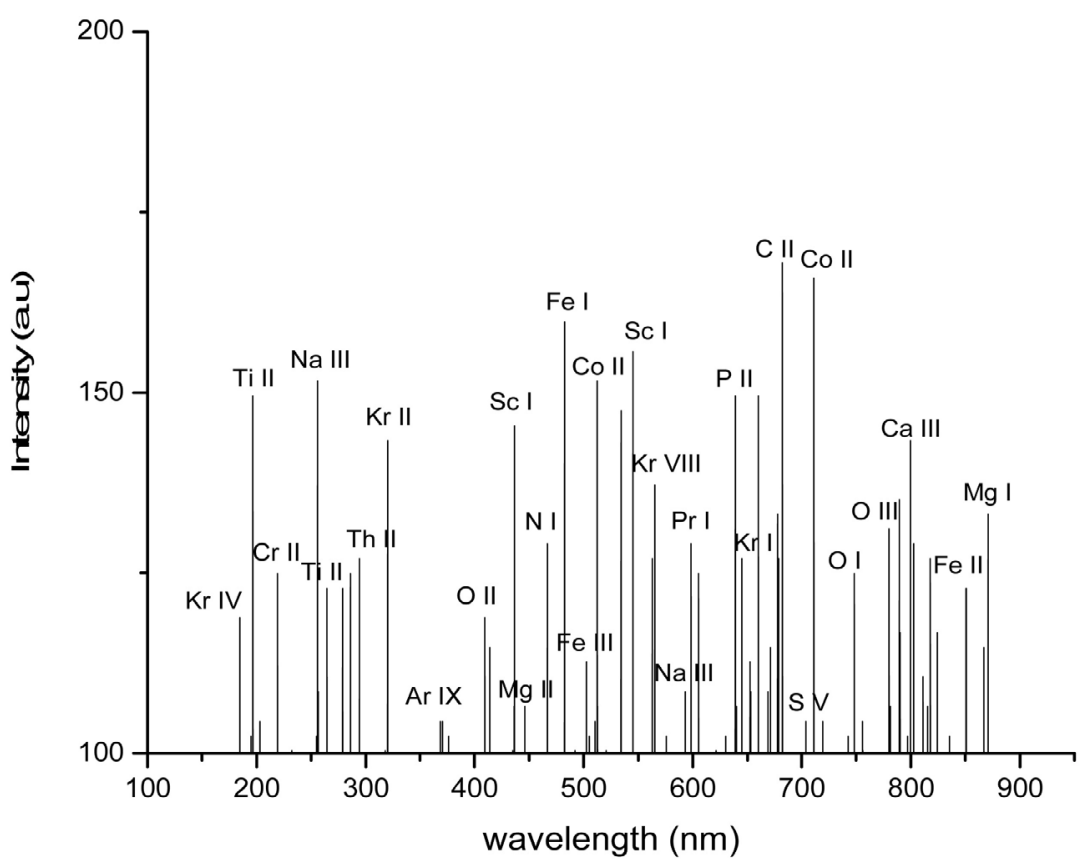

Figure 2. LIBS emission spectrum of sample (1).

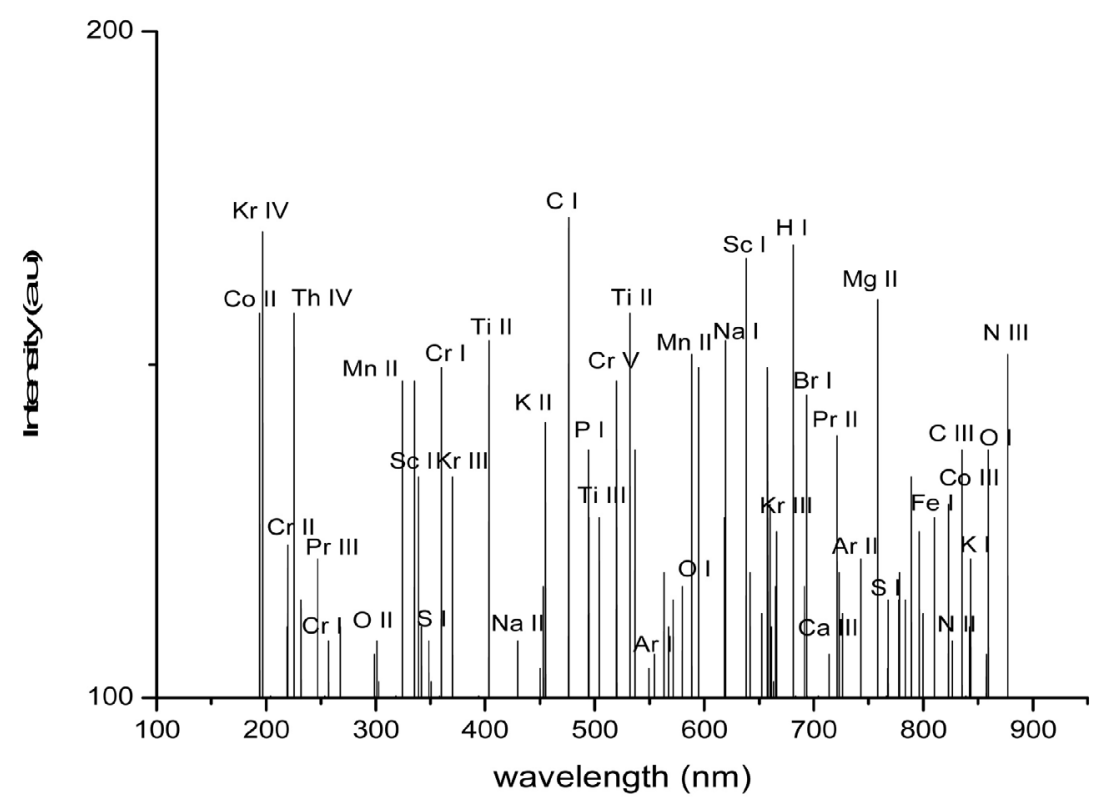

Figure 3. LIBS emission spectrum of sample (2). 


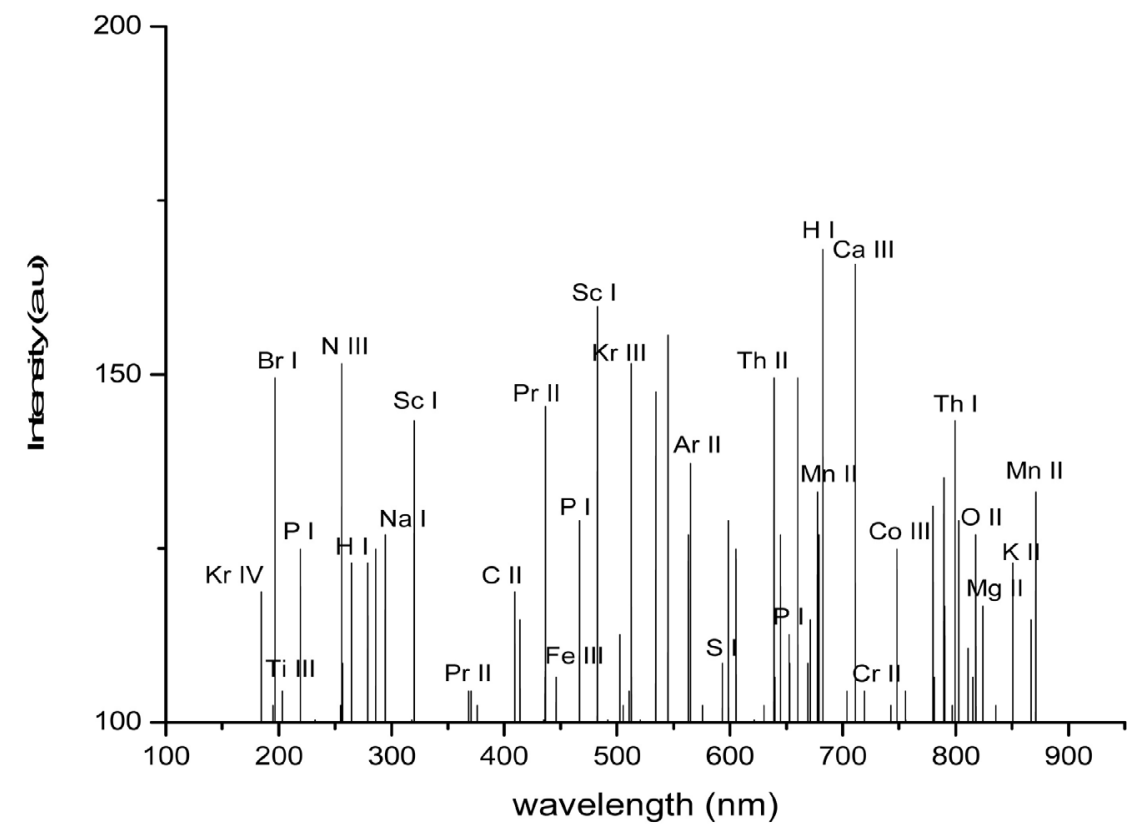

Figure 4. LIBS emission spectrum of sample (3).

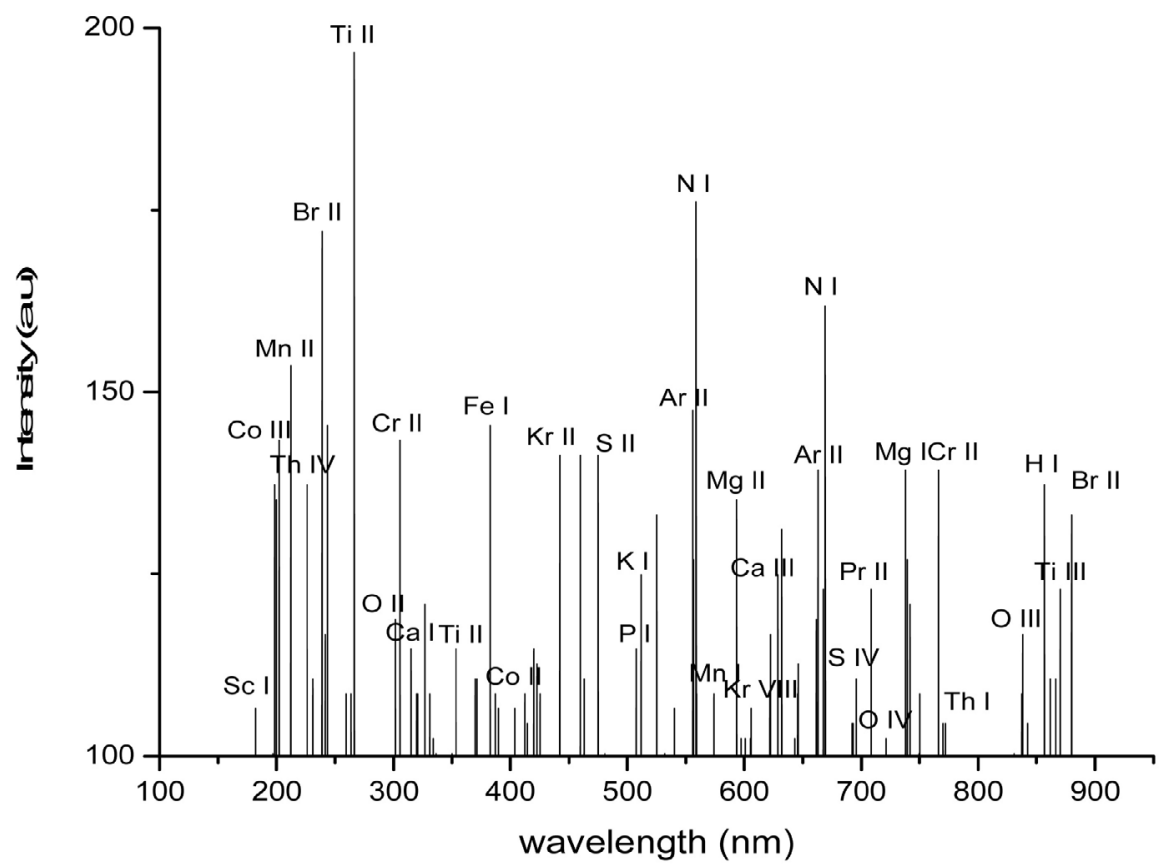

Figure 5. LIBS emission spectrum of sample (4).

( $\mathrm{Ti}, \mathrm{Br}, \mathrm{Ar}, \mathrm{Th}, \mathrm{Kr}, \mathrm{Sc}$ and $\mathrm{Pr}$ ).

Interestingly the elements $\mathrm{Th}, \mathrm{Pr}, \mathrm{Kr}$, and Sc have not been observed in any of the previous studies undertaken on Acacia nilotica using the conventional (AAS or ICP) techniques. These elements and their ions are reported here for the first time in the elemental analysis of Acacia nilotica using LIBS, this adds to the many advantages of this technique, compared to other conventional techniques mentioned above. Sample of Acacia nilotica showed the presence of heavy metals like $\mathrm{Fe}, \mathrm{Cr}, \mathrm{Pr}$ and Th which may hinder its application in food and pharmaceutical formulation. 
Table 2. The analyzed data of Acacia nilotica (sunt) collected from different locations after irradiation by laser energy of $80 \mathrm{~mJ}$.

\begin{tabular}{|c|c|c|c|c|c|}
\hline \multirow{2}{*}{ Element } & \multirow{2}{*}{$\lambda(\mathrm{nm})$} & \multicolumn{4}{|c|}{ Emission intensity (a.u) } \\
\hline & & $\left(\mathrm{S}_{11}\right)$ & $\left(\mathrm{S}_{12}\right)$ & $\left(\mathrm{S}_{13}\right)$ & $\left(\mathrm{S}_{14}\right)$ \\
\hline \multirow{7}{*}{ Fe I } & 217.0590 & & & 121.0267 & 110.7591 \\
\hline & 228.7649 & & 106.2807 & 159.9672 & \\
\hline & 314.4824 & & & 137.4112 & 135.4997 \\
\hline & 345.0688 & & 128.9459 & 133.2605 & \\
\hline & 401.3327 & 119.3883 & 135.4997 & & \\
\hline & 498.3784 & 115.2375 & 137.4112 & 112.7252 & 151.8842 \\
\hline & 516.5037 & & 125.1774 & 104.2053 & 127.4713 \\
\hline \multirow{7}{*}{ Fe II } & 185.7174 & 111.0868 & & 108.7930 & \\
\hline & 215.7110 & & 104.9699 & 121.0267 & 110.7591 \\
\hline & 258.5961 & & 110.7591 & 141.6166 & \\
\hline & 510.0844 & & 125.1774 & 112.9437 & \\
\hline & 633.5628 & 116.8760 & 121.3544 & & 110.7591 \\
\hline & 684.1625 & 103.0038 & & 149.6450 & \\
\hline & 797.4455 & 149.6450 & & 125.5051 & 158.0010 \\
\hline \multirow{7}{*}{ Fe III } & 364.3269 & 118.7329 & 114.9098 & 135.4997 & 110.7591 \\
\hline & 512.7276 & & 125.1774 & 127.4713 & \\
\hline & 538.7827 & & 129.0005 & 116.8760 & \\
\hline & 596.5570 & & 114.5821 & & \\
\hline & 618.4584 & & 129.0005 & & \\
\hline & 731.7414 & & 123.3205 & 122.6990 & 110.7591 \\
\hline & 823.1230 & 165.4833 & & & 112.9437 \\
\hline \multirow{5}{*}{$\mathrm{Na} \mathrm{I}$} & 249.1559 & & & 131.2943 & 114.9098 \\
\hline & 261.2394 & 103.0038 & 110.7591 & 165.4287 & \\
\hline & 355.2643 & & 126.8159 & 137.4112 & \\
\hline & 589.4944 & & 104.6422 & & 119.0606 \\
\hline & 691.7147 & 148.0611 & 104.3691 & 104.3145 & 141.9442 \\
\hline \multirow{5}{*}{$\mathrm{Na} I I$} & 242.7364 & & & 131.2943 & \\
\hline & 254.8200 & 135.4997 & 116.8760 & 152.2119 & 149.6450 \\
\hline & 308.0630 & 104.6422 & & 122.9929 & \\
\hline & 316.3705 & & 126.8159 & 158.2195 & 135.4997 \\
\hline & 519.1470 & & & 104.2053 & 127.4713 \\
\hline
\end{tabular}




\section{Continued}

\begin{tabular}{|c|c|c|c|c|c|}
\hline \multirow{6}{*}{$\mathrm{Na} I I I$} & 203.0875 & 106.6084 & 104.9699 & 141.6166 & 139.0497 \\
\hline & 323.9227 & 137.7389 & & 125.1774 & 113.2168 \\
\hline & 395.6685 & 125.1774 & 118.7329 & 135.1720 & \\
\hline & 590.8929 & & 114.9098 & & 119.0606 \\
\hline & 652.4433 & 129.3282 & 127.1436 & & 113.5991 \\
\hline & 713.6161 & 162.2610 & & & 138.0666 \\
\hline \multirow{4}{*}{$\mathrm{Ca} \mathrm{I}$} & 272.1901 & & 103.0038 & 108.7930 & 122.9929 \\
\hline & 616.9480 & 143.8558 & 129.0005 & 127.7990 & \\
\hline & 720.0355 & & & 163.7902 & 111.0868 \\
\hline & 734.7623 & 119.7160 & 123.3205 & 112.9437 & 119.0606 \\
\hline \multirow{3}{*}{ Ca II } & 423.2341 & 119.0606 & 103.0038 & 108.7930 & 119.0606 \\
\hline & 608.6406 & & 126.8159 & 119.0606 & \\
\hline & 757.0413 & 164.1179 & 115.2375 & & \\
\hline \multirow{4}{*}{$\mathrm{Ca}$ III } & 199.3114 & & 129.9836 & 114.9098 & \\
\hline & 281.6303 & 120.8083 & & 136.7558 & 139.3773 \\
\hline & 508.1963 & & 137.4112 & 112.9437 & \\
\hline & 535.0066 & & 129.0005 & 116.8760 & 133.2605 \\
\hline \multirow{7}{*}{$\mathrm{Mg} \mathrm{I}$} & 265.7707 & 120.8083 & 114.9098 & 118.8421 & \\
\hline & 548.6006 & & & 192.7362 & 121.0267 \\
\hline & 631.6748 & 116.8760 & 121.3544 & & 110.7591 \\
\hline & 748.7338 & 135.4997 & & & 113.2714 \\
\hline & 781.2083 & 114.9098 & 111.0868 & & \\
\hline & 805.3753 & 141.2889 & 153.8503 & 129.3282 & 112.9437 \\
\hline & 847.2900 & 129.3282 & & 125.1774 & 125.1774 \\
\hline \multirow{5}{*}{ Mg II } & 355.2643 & & 126.8159 & 108.5745 & \\
\hline & 427.0102 & & 103.0038 & 108.7930 & \\
\hline & 545.2021 & 141.6166 & & 192.7362 & 121.0267 \\
\hline & 787.6277 & & 111.0868 & 112.9437 & \\
\hline & 811.4171 & 137.0835 & 153.8503 & 126.7613 & \\
\hline \multirow{5}{*}{ Mg III } & 183.0741 & 111.0868 & & 165.4287 & 135.4997 \\
\hline & 425.1221 & 119.0606 & 103.0038 & 108.7930 & 119.0606 \\
\hline & 562.5721 & 133.2605 & 139.0479 & 112.9437 & 125.5051 \\
\hline & 692.4700 & 148.0611 & 104.3691 & & 141.9442 \\
\hline & 704.5535 & 143.5281 & & 123.3205 & 133.2605 \\
\hline
\end{tabular}




\begin{tabular}{|c|c|c|c|c|c|}
\hline \multirow{4}{*}{ K I } & 297.1123 & 137.7389 & 165.7564 & 116.8760 & 113.2168 \\
\hline & 690.9595 & 141.9442 & 104.3691 & 104.3145 & 106.3353 \\
\hline & 710.9729 & 162.2610 & 114.9098 & 106.9361 & 138.0666 \\
\hline & 785.7396 & 137.7389 & & 112.9437 & \\
\hline \multirow{3}{*}{ K II } & 368.8582 & 125.1774 & 121.0267 & 141.6166 & \\
\hline & 380.9418 & 115.2375 & & 118.8421 & \\
\hline & 579.1870 & 103.0038 & 108.7930 & & 112.6160 \\
\hline \multirow{5}{*}{ K III } & 334.1181 & & 111.0868 & & \\
\hline & 348.0897 & & & 133.2605 & 116.5483 \\
\hline & 388.4940 & & & 139.0497 & \\
\hline & 457.5966 & 120.8083 & & 112.7252 & 112.6160 \\
\hline & 767.2367 & 104.6422 & 106.9361 & 114.5821 & 145.7673 \\
\hline \multirow{7}{*}{ S I } & 540.2932 & 119.0606 & & 192.7362 & 121.0267 \\
\hline & 558.0408 & & 125.1774 & 125.8328 & 125.5051 \\
\hline & 595.8018 & & 114.5821 & 119.0606 & \\
\hline & 673.9671 & 123.3205 & 112.9437 & 112.9437 & \\
\hline & 724.1892 & & 110.8137 & 163.7902 & 111.0868 \\
\hline & 792.9142 & & 126.7613 & 108.7930 & \\
\hline & 866.5482 & 108.7930 & 139.3773 & & 127.1436 \\
\hline \multirow{7}{*}{ S II } & 361.6836 & & 110.7591 & & \\
\hline & 405.8640 & 119.3883 & 135.4997 & & \\
\hline & 500.6441 & 106.9361 & & & 164.1179 \\
\hline & 522.9231 & 131.2943 & 109.1206 & 188.2577 & 135.4997 \\
\hline & 536.8947 & 114.9098 & 129.0005 & 116.8760 & \\
\hline & 687.9386 & 141.9442 & & 155.8164 & 119.0606 \\
\hline & 740.4264 & 112.9437 & 137.4112 & & \\
\hline \multirow{3}{*}{ S III } & 252.1768 & 135.4997 & 116.8760 & & 114.9098 \\
\hline & 337.5166 & 161.9333 & 104.9699 & & \\
\hline & 632.4300 & 143.5281 & & 106.2807 & 110.7591 \\
\hline \multirow{5}{*}{ C I } & 473.4562 & 103.0038 & 133.2605 & 165.4287 & 147.7334 \\
\hline & 529.3425 & 114.9098 & 116.5483 & & \\
\hline & 568.9915 & 139.3773 & 112.6160 & 129.3282 & 121.0267 \\
\hline & 579.9422 & & & 112.6160 & 103.0038 \\
\hline & 763.4606 & 136.1551 & 110.8137 & 163.7902 & 111.0868 \\
\hline
\end{tabular}




\begin{tabular}{|c|c|c|c|c|c|}
\hline \multirow{4}{*}{ C II } & 359.0404 & & 125.1774 & 112.9437 & \\
\hline & 625.2554 & 110.7591 & 129.0005 & 106.6084 & 133.2605 \\
\hline & 663.7716 & 122.9929 & & & 119.0606 \\
\hline & 803.1097 & 141.2889 & 153.8503 & 129.3282 & \\
\hline \multirow{4}{*}{ C III } & 218.1919 & 131.2943 & 109.1206 & 151.6056 & 135.4997 \\
\hline & 794.8023 & 149.6450 & & 108.7930 & \\
\hline & 853.709 & 114.9098 & 116.8760 & 104.6422 & \\
\hline & 880.5197 & 137.7389 & 139.3773 & & 112.9437 \\
\hline \multirow{5}{*}{ N I } & 493.4695 & 110.7591 & 103.0038 & 106.6084 & 135.4997 \\
\hline & 672.0790 & 106.6084 & 117.2037 & 112.9437 & \\
\hline & 765.3487 & 104.6422 & & 114.5821 & \\
\hline & 789.8933 & & 111.0868 & 112.9437 & \\
\hline & 870.3243 & 108.7930 & & 121.0267 & \\
\hline \multirow{6}{*}{ N II } & 384.7179 & 120.8083 & & 118.8421 & \\
\hline & 502.5322 & 106.9361 & & 176.0240 & 164.1179 \\
\hline & 531.9857 & 114.9098 & 116.5483 & 116.8760 & 133.2605 \\
\hline & 593.1585 & & 126.8159 & 119.0606 & 119.0606 \\
\hline & 683.4073 & 103.0038 & & 149.6450 & \\
\hline & 700.0222 & & 151.8842 & 155.8164 & 119.0606 \\
\hline \multirow{4}{*}{ N III } & 184.5846 & 111.0868 & 104.9699 & 108.7930 & 104.9699 \\
\hline & 471.1905 & 103.0038 & & 136.8651 & 145.7673 \\
\hline & 489.6934 & & & 171.8732 & \\
\hline & 644.5135 & 110.7591 & 129.0005 & 106.6084 & 133.2605 \\
\hline \multirow{3}{*}{ O I } & 613.9271 & 143.8558 & 127.1436 & 141.6166 & 106.6084 \\
\hline & 777.4322 & & & 122.6990 & 110.7591 \\
\hline & 840.8707 & 121.0267 & 108.7930 & 141.6166 & \\
\hline \multirow{6}{*}{ O II } & 296.469 & & & 116.8760 & \\
\hline & 394.9133 & 125.1774 & 118.7329 & 135.1720 & 110.7591 \\
\hline & 444.7578 & & & 145.7673 & 110.7591 \\
\hline & 638.094 & 111.4145 & & 106.2807 & \\
\hline & 736.6503 & 119.7160 & 103.0038 & 106.9361 & 122.6652 \\
\hline & 762.7054 & 104.6422 & & 114.5821 & \\
\hline
\end{tabular}




\section{Continued}

\begin{tabular}{|c|c|c|c|c|c|}
\hline \multirow{9}{*}{ O III } & 319.3913 & & 105.2976 & 158.2195 & 135.4997 \\
\hline & 351.4882 & & 128.9459 & & 116.5483 \\
\hline & 394.5257 & 125.1774 & 118.7329 & 135.1720 & \\
\hline & 650.9329 & 129.3282 & 127.1436 & & 113.5991 \\
\hline & 673.5895 & 123.3205 & 117.2037 & 112.9437 & \\
\hline & 729.4757 & 119.3883 & 110.8137 & 112.9437 & \\
\hline & 749.8667 & 135.4997 & 149.6450 & & 113.2714 \\
\hline & 809.5290 & 137.0835 & & 125.5051 & 158.0010 \\
\hline & 817.0812 & 161.9333 & 110.4314 & 110.7591 & 127.1436 \\
\hline \multirow{5}{*}{ Cr I } & 194.0248 & & & 114.9098 & 135.4997 \\
\hline & 212.1501 & 106.6084 & 104.9699 & & \\
\hline & 234.4291 & 114.9098 & 120.6990 & 153.8503 & 147.7334 \\
\hline & 346.9569 & & & 133.2605 & 116.5483 \\
\hline & 456.3637 & 119.3883 & & 112.7252 & 104.6422 \\
\hline \multirow{5}{*}{ Cr II } & 253.6872 & 135.4997 & 116.8760 & 152.2119 & 149.6450 \\
\hline & 275.9662 & 104.6422 & & 165.4287 & 147.7334 \\
\hline & 386.6059 & 120.8083 & & 139.0497 & \\
\hline & 554.2647 & 119.0606 & 125.1774 & 120.6990 & 108.7930 \\
\hline & 572.7676 & & & 111.0868 & \\
\hline \multirow{2}{*}{$\mathrm{Cr} \mathrm{V}$} & 637.7165 & 123.3205 & & 106.2807 & \\
\hline & 731.3638 & 119.3883 & 123.3205 & 112.9437 & 149.6450 \\
\hline \multirow{4}{*}{ Ti I } & 259.3513 & & 110.7591 & & \\
\hline & 370.7463 & 104.6422 & 121.0267 & 114.9098 & 156.0349 \\
\hline & 478.3651 & & 133.2605 & 104.6422 & \\
\hline & 562.1945 & 139.3773 & 139.0479 & 112.9437 & 125.5051 \\
\hline \multirow{4}{*}{ Ti II } & 229.1426 & 114.9098 & 106.2807 & 159.9672 & \\
\hline & 299.0004 & & 103.0038 & 136.7558 & 139.3773 \\
\hline & 430.7863 & 103.1130 & & 163.7902 & 111.0868 \\
\hline & 521.0350 & 131.2943 & & 188.2577 & 135.4997 \\
\hline \multirow{3}{*}{ Ti III } & 350.7330 & & & 133.2605 & 116.5483 \\
\hline & 755.1532 & 143.5281 & 111.0868 & 119.0606 & 104.6422 \\
\hline & 829.9200 & 112.9437 & 114.9098 & 110.7591 & 119.0606 \\
\hline \multirow{4}{*}{ Br I } & 238.582 & 119.0606 & 103.0038 & 103.0038 & 147.4047 \\
\hline & 518.769 & & & 104.2053 & 127.4713 \\
\hline & 668.302 & 122.9929 & & 125.1774 & 106.3353 \\
\hline & 813.305 & 161.9333 & 110.4314 & 126.7613 & 127.1436 \\
\hline
\end{tabular}




\section{Continued}

\begin{tabular}{|c|c|c|c|c|c|}
\hline \multirow{2}{*}{ Br II } & 417.9475 & & 103.0038 & 106.9361 & 122.6652 \\
\hline & 797.8231 & 149.6450 & & 108.7930 & \\
\hline \multirow{6}{*}{ Ar I } & 375.2776 & & & 121.0267 & 156.0349 \\
\hline & 437.9609 & 104.9699 & & 135.4997 & \\
\hline & 526.6992 & & 109.1206 & 151.6056 & \\
\hline & 556.1528 & & 125.1774 & 125.8328 & 108.7930 \\
\hline & 565.2154 & 139.3773 & 110.7591 & 112.9437 & \\
\hline & 654.7090 & 129.3282 & 127.1436 & 127.7990 & 113.5991 \\
\hline \multirow{6}{*}{ Ar II } & 380.9418 & 120.8083 & & 118.8421 & 104.6422 \\
\hline & 523.6783 & 131.2943 & 109.1206 & 151.6056 & 135.4997 \\
\hline & 538.4051 & & 129.0005 & 135.4997 & 106.6084 \\
\hline & 684.5402 & 143.5281 & & 149.6450 & 103.3315 \\
\hline & 783.8516 & 114.9098 & & 131.2943 & \\
\hline & 879.0093 & 121.0267 & & & 112.9437 \\
\hline \multirow{3}{*}{ Ar IV } & 244.6246 & 111.0868 & & 131.2943 & 104.9699 \\
\hline & 464.7712 & & 103.1130 & 176.0240 & 110.7591 \\
\hline & 803.4873 & 141.2889 & 153.8503 & 129.3282 & \\
\hline \multirow{5}{*}{ Th I } & 373.0119 & 120.8083 & & 118.8421 & 156.0349 \\
\hline & 585.6069 & & 104.6422 & 104.6422 & 147.7334 \\
\hline & 721.1683 & & 103.1130 & 163.7902 & 111.0868 \\
\hline & 764.2159 & 104.6422 & & 114.5821 & 145.7673 \\
\hline & 778.5650 & 108.7930 & 111.0868 & 122.6990 & 110.7591 \\
\hline \multirow{6}{*}{ Th II } & 376.7880 & & & 121.0267 & \\
\hline & 478.3651 & & 133.2605 & 104.6422 & \\
\hline & 537.2723 & & 129.0005 & 116.8760 & \\
\hline & 594.6690 & & 114.5821 & & 119.0606 \\
\hline & 621.1017 & 110.7591 & & 106.6084 & 133.2605 \\
\hline & 858.6183 & 114.9098 & & 104.6422 & \\
\hline \multirow{5}{*}{ Kr III } & 213.6605 & 106.6084 & 104.9699 & & 110.7591 \\
\hline & 251.0493 & 135.4997 & 116.8760 & 152.2119 & 114.9098 \\
\hline & 285.0288 & & & 136.7558 & 139.3773 \\
\hline & 371.1239 & & 105.2976 & 114.9098 & 156.0349 \\
\hline & 452.3100 & & & 112.7252 & 104.6422 \\
\hline
\end{tabular}




\section{Continued}

\begin{tabular}{|c|c|c|c|c|c|}
\hline \multirow{4}{*}{ Mn II } & 255.4424 & 135.4997 & 116.8760 & 152.2119 & 149.6450 \\
\hline & 313.7272 & & & 137.4112 & \\
\hline & 320.5242 & 137.7389 & 105.2976 & 125.1774 & 135.4997 \\
\hline & 635.4509 & 116.8760 & 121.3544 & 106.2807 & 110.7591 \\
\hline \multirow{4}{*}{ Sc I } & 460.9951 & & & 112.7252 & 110.7591 \\
\hline & 474.5890 & 103.0038 & 133.2605 & 104.6422 & 145.7673 \\
\hline & 544.0693 & 141.6166 & & 192.7362 & \\
\hline & 632.0524 & 116.8760 & 121.3544 & & 110.7591 \\
\hline \multirow{4}{*}{ Pr II } & 513.4828 & & 125.1774 & 127.4713 & \\
\hline & 550.8662 & & & 192.7362 & 121.0267 \\
\hline & 587.8720 & & 104.6422 & & \\
\hline & 594.6690 & 129.3282 & 114.5821 & & 119.0606 \\
\hline \multirow{3}{*}{ Co III } & 667.5477 & 122.9929 & & 125.1774 & 106.3353 \\
\hline & 743.8249 & 112.9437 & 111.0868 & 163.7902 & 104.6422 \\
\hline & 794.4247 & 149.6450 & 111.0868 & 108.7930 & \\
\hline \multirow{4}{*}{ P I } & 274.8334 & 104.6422 & & & \\
\hline & 342.4255 & & 128.9459 & 104.6968 & \\
\hline & 474.9666 & 103.0038 & 133.2605 & 104.6422 & 145.7673 \\
\hline & 551.6215 & 136.1551 & 125.1774 & 120.6990 & 108.7930 \\
\hline \multirow{7}{*}{ H I } & 373.7672 & & 121.0267 & 114.9098 & 156.0349 \\
\hline & 393.0253 & & 118.7329 & & \\
\hline & 410.395 & 119.3883 & 113.5991 & & 111.0868 \\
\hline & 434.184 & 103.1130 & & 135.4997 & 117.2037 \\
\hline & 486.0502 & 103.0038 & 133.2605 & 171.8732 & \\
\hline & 656.5970 & 202.2938 & 202.6215 & 131.6220 & 110.7591 \\
\hline & 832.5633 & & & 110.7591 & 135.4997 \\
\hline
\end{tabular}

\section{CONCLUSIONS}

Sample of Acacia nilotica showed the presence of heavy metals like Fe, Cr, Pr and Th which may hinder its application in food and pharmaceutical formulation and the elemental composition of Gum Arabic by LIBS technique enabled observing elements, such as $\mathrm{Br}, \mathrm{Ti}$ and $\mathrm{Ar}$, to be reported for the first time.

\section{CONFLICTS OF INTEREST}

The authors declare no conflicts of interest regarding the publication of this paper. 


\section{REFERENCES}

1. Cremers, D.A. and Radziemski, L.J. (2006) Appendix D: Major LIBS References. Handbook of Laser-Induced Breakdown Spectroscopy, 271-273. https://doi.org/10.1002/0470093013.app4

2. Miziolek, A.W., Palleschi, V. and Schechter, I., Eds. (2006) Laser Induced Breakdown Spectroscopy. Cambridge University Press, Cambridge. https://doi.org/10.1017/CBO9780511541261

3. Ahmed, R. and Baig, M.A. (2009) A Comparative Study of Single and Double Pulse Laser Induced Breakdown Spectroscopy. Journal of Applied Physics, 106, 033307. https://doi.org/10.1063/1.3190516

4. Ahmed, R. and Baig, M.A. (2010) On the Optimization for Enhanced Dual-Pulse Laser-Induced Breakdown Spectroscopy. IEEE Transactions on Plasma Science, 38, 2052-2055. https://doi.org/10.1109/TPS.2010.2050784

5. Palleschi, A. and Salvetti, E.T. (2002) Spectrochim. Acta, Part B, 57, 339. (F.C. DeLucia Jr, J.L. Gottfried, Mater., 2011)

6. Wisbrun, R., Schechter, I., Niessner, R., Schroeder, H. and Kompa, K.L. (1994) Detector for Trace Elemental Analysis of Solid Environmental Samples by Laser Plasma Spectroscopy. Analytical Chemistry, 66, 2964-2975. https://doi.org/10.1021/ac00090a026

7. Cáceres, J.O., López, J.T., Telle, H.H. and Ureña, A.G. (2001) Quantitative Analysis of Trace Metal Ions in Ice Using Laser-Induced Breakdown Spectroscopy. Spectrochimica Acta Part B: Atomic Spectroscopy, 56, 831-838. https://doi.org/10.1016/S0584-8547(01)00173-2

8. Wickens, G.E. (1995) Role of Acacia Species in the Rural Economy of Dry Africa and the Near East (No. 27). Food \& Agriculture Org.

9. (2016) Acacia nilotica (Gum Arabic Tree). Invasive Species Compendium. Centre for Agriculture and Biosciences International. Retrieved 24 January.

10. Smolinske, S.C. (1992) CRC Handbook of Food, Drug, and Cosmetic Excipients. CRC Press.

11. Renard, D., Lavenant-Gourgeon, L., Ralet, M.C. and Sanchez, C. (2006) Acacia Senegal Gum: Continuum of Molecular Species Differing by Their Protein to Sugar Ratio, Molecular Weight, and Charges. Biomacromolecules, 7, 2637-2649. https://doi.org/10.1021/bm060145j

12. Anderson, D.M.W., Douglas, D.B., Morrison, N.A. and Weiping, W. (1990) Specifications for Gum Arabic (Acacia Senegal); Analytical Data for Samples Collected between 1904 and 1989. Food Additives \& Contaminants, 7, 303-321. https://doi.org/10.1080/02652039009373896

13. Bulajic, D., et al. (2002) A Procedure for Correcting Self-Absorption in Calibration Free-Laser Induced Breakdown Spectroscopy. Spectrochimica Acta Part B: Atomic Spectroscopy, 57, 339-353. https://doi.org/10.1016/S0584-8547(01)00398-6 